\title{
Ciudades y luces a principios de la Edad del Bronce Antiguo. Sobre las primeras lámparas de aceite en el Levante meridional
}

\author{
(4) Nimrod Getzov \\ Autoridad de Antigüedades de Israel, Israel \\ lanir Milevski \\ Autoridad de Antigüedades de Israel, Israel / Programa “Raíces”, Ministerio de Ciencia, \\ Tecnología e Innovación Productiva, Argentina
}

Fecha de recepción: 25 de julio de 2021. Fecha de aceptación: 18 de agosto de 2021.

\section{Resumen}

En este artículo se analizarán las primeras lámparas de aceite de arcilla en forma de cuencos halladas en las excavaciones de Ein Zippori, en la Baja Galilea, y se pasará revista de los mismos hallazgos en varios sitios de la Edad del Bronce Antiguo IB en el Levante meridional. Se trata del comienzo de la aparición generalizada de estas lámparas en los hallazgos arqueológicos de la región del Levante meridional, por lo menos en lo que hoy día es Israel y los territorios de la Autoridad Palestina, a la luz de la primera urbanización de la región y de un cambio en la organización social y económica de estas comunidades.

Palabras clave: Bronce Antiguo I, Ein Zippori, Levante meridional, lámparas de aceite, división del trabajo, urbanización

Cities and Lights in the Early Bronze Age. On the First Oil Lamps in the Southern Levant

\footnotetext{
Abstract

This article will analyze the firs oil lamps, bowl-shaped clay containers, found at Ein Zippori excavations in the Lower Galilee, and will review the same findings at various Early Bronze Age (EB) sites in the southern Levant. The EB IB is the beginning of the widespread appearance of these lamps in
} 
archaeological finds in the southern Levantine sites, at least in what is now Israel and the territories of the Palestinian Authority in light of the first urbanization of the region, and a change in the social and economic organization of these communities.

Keywords: Early Bronze Age I, Ein Zippori, southern Levant, oil lamps, division of labor, urbanization

\section{Introducción}

En varios sitios de la Edad del Bronce Antiguo IB se han encontrado muchas lámparas de aceite y al parecer éste fue el comienzo de la aparición generalizada de lámparas en los hallazgos arqueológicos del Levante meridional, por lo menos en lo que hoy día es Israel y los territorios de la Autoridad Palestina (Fig. 1). Nuestra investigación sobre el tema comenzó a partir de las excavaciones en Ein Zippori, en la Baja Galilea, pero hemos descubierto que el fenómeno es mucho más amplio. Tenemos entonces que a fines del IV milenio a.C. se han dado las bases económicas y sociales para el uso generalizado de lámparas de aceite. En este artículo pretenderemos dar una explicación a dicho fenómeno.

En los años 2011-2013, la Autoridad de Antigüedades de Israel llevó a cabo largas excavaciones de rescate en el sitio de Ein Zippori (Milevski y Getzov, 2014; Milevski et al., 2014; Yaroshevich, 2016). El sitio se extiende al sur del manantial de Ein Zippori, en el borde de un pequeño valle y al pie de la colina denominada Givat Rabi (Fig. 2). Los restos del asentamiento de la Edad del Bronce Antiguo se extienden hasta la cima de Givat Rabi, en su vertiente norte, y en una ligera pendiente hacia el borde del valle. El sitio contiene los restos de asentamientos prehistóricos y protohistóricos cuya investigación comenzó ya en el siglo anterior: un relevamiento realizado por Z. Gal (2002), una excavación limitada realizada por D. Kaufman de la Universidad de Haifa (sin publicar), una excavación limitada realizada por O. Barzilai (Barzilai et al., 2014) y un relevamiento por ampliación de la carretera 79, conducido por O. Marder y H. Khalaily, seguida de las excavaciones detalladas aquí.

Se excavó un área de aproximadamente 0,75 hectáreas (aprox. 300 cuadrículas de 5 x $5 \mathrm{~m}$ ). En algunos lugares, la excavación llegó hasta el suelo virgen o hasta la roca. En la base de las acumulaciones se encuentran restos del período Neolítico y del período Calcolítico temprano. Los restos del Bronce Antiguo se encuentran por encima de los estratos de los períodos Neolítico y Calcolítico en la mayor parte de las áreas de excavación. 


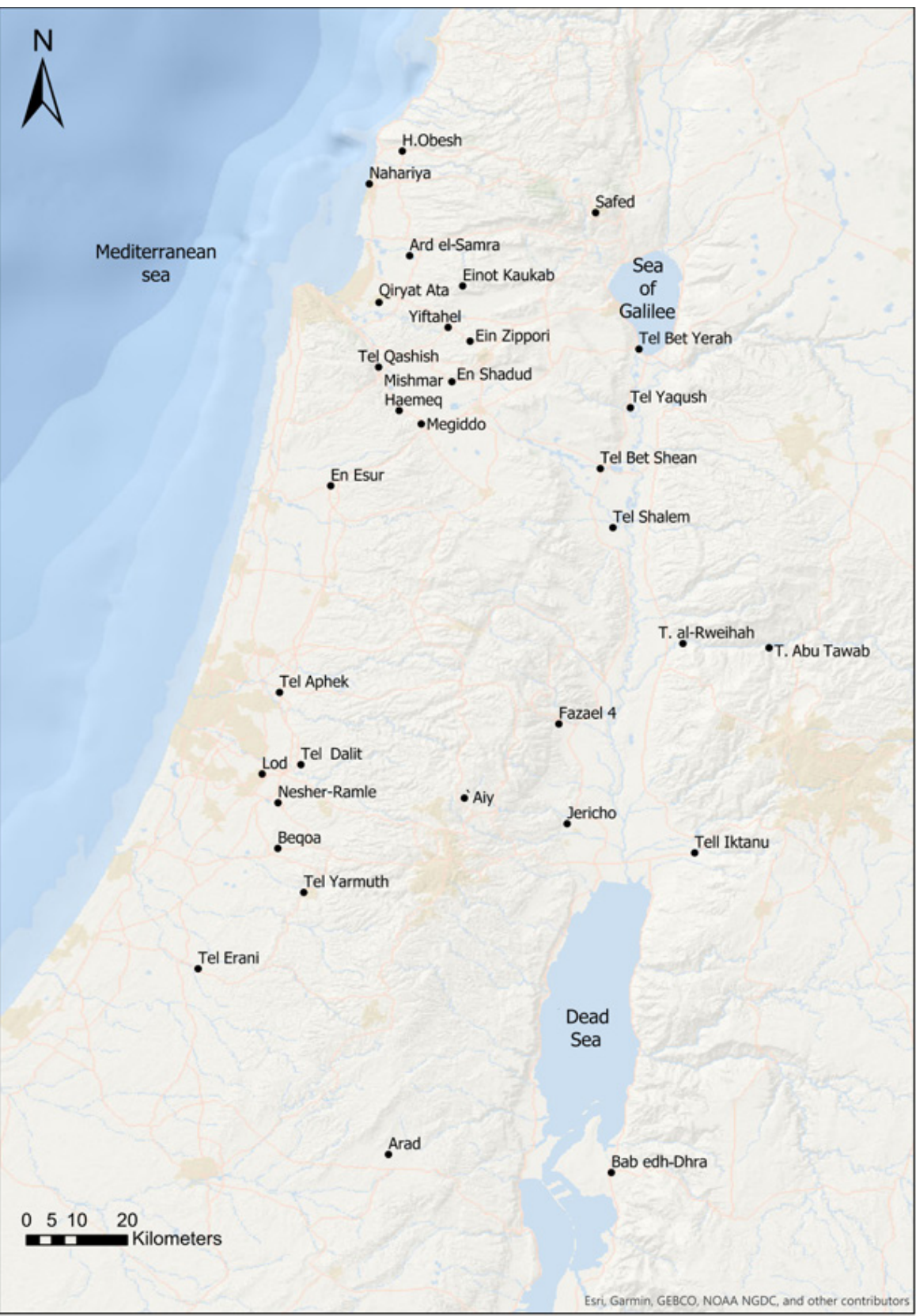

Fig. 1. Mapa con la ubicación de los sitios mencionados en el texto (producción Atalya Fadida, basado en el programa ESRI). 


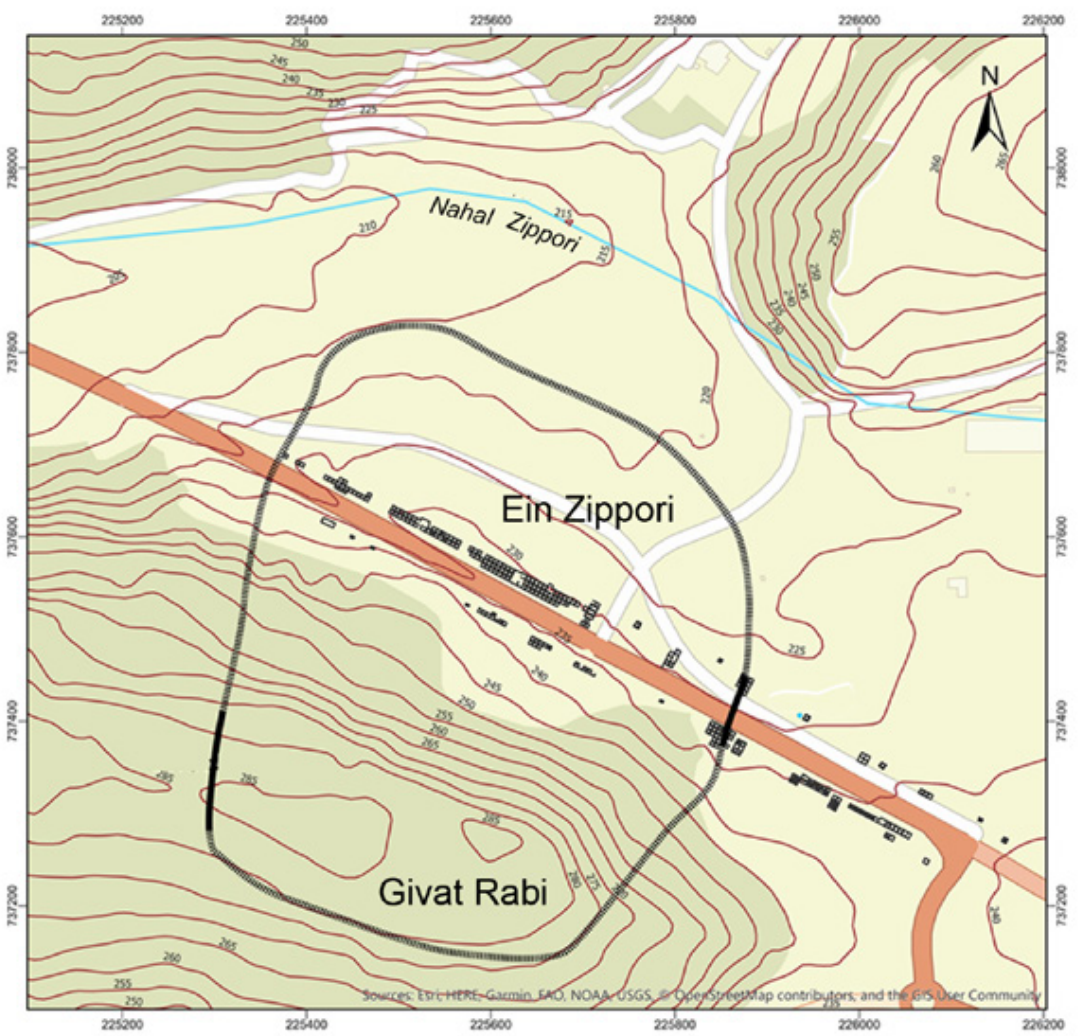

Fig. 2. Ein Zippori, plano de las áreas de excavación (producción Atalya Fadida, cortesía IAA).

\section{Edad del Bronce Antiguo IB}

Los cimientos de los edificios eran de piedra. En el eje central de las salas se encontraron losas de piedra, sobre las cuales seguramente se colocaban pilares. Las esquinas de los edificios eran redondeadas, característica de este período en toda la región. Durante la historia del asentamiento se construyeron cuartos adicionales que se apoyaban contra los muros de los edificios anteriores, pero también se levantaron nuevas paredes que ocupaban y dividían los espacios que quedaban entre los distintos edificios (Fig. 3). 


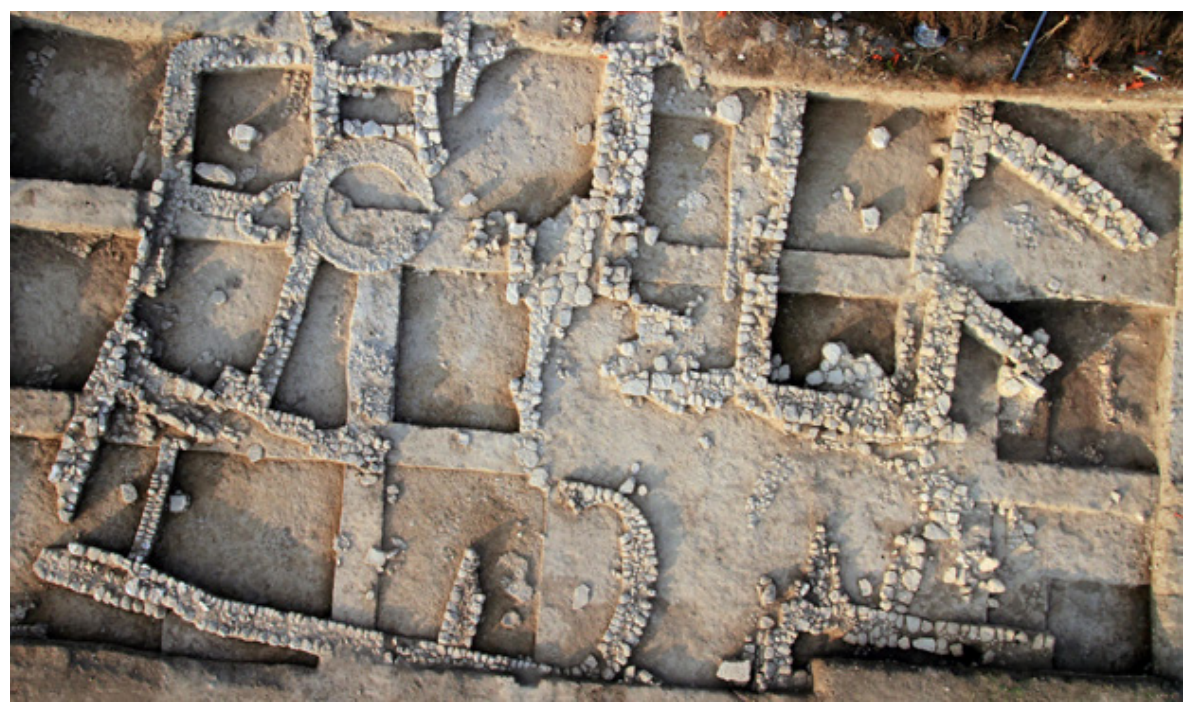

Fig. 3. Ein Zippori, edificios del Área B, vista aérea (cortesía IAA).

\begin{tabular}{cc}
\hline Período & Años a.C. \\
\hline BA IA & $3700-3500$ \\
\hline BA IB & $3500-3100$ \\
\hline BA II & $3100-2800$ \\
\hline BA III & $2800-2500$ \\
\hline
\end{tabular}

Tabla 1. Cronología de la Edad del Bronce Antiguo (BA) (Regev et al., 2012a; 2012b; 2019; ver también Rosenzweig, 2020).

En el límite oriental de los restos de la Edad del Bronce Antiguo se encontró un muro de 1,9 m de ancho (Fig. 4). Durante la excavación de este muro en las áreas D3 y N5 quedó expuesta una base construida de tres hileras de piedras medianas y probablemente una pared de ladrillos encima de ella (Fig. 4: 1). En el mismo se encontró una puerta poterna de $80 \mathrm{~cm}$ de ancho (Fig. 4:3) similar a muchas puertas encontradas en los muros de etapas posteriores de la Edad del Bronce Antiguo. Frente al muro y paralela al mismo fue encontrada parte de una habitación con una esquina redondeada que es el sello distintivo de esta fase (Fig. 4: 2), datada por $\mathrm{C}^{14}$ entre el 3300 y el 3100 cal. BC por J. Regev y E. Boaretto del Instituto Weizmann. En otra parte del muro, al sur de la aquí descrita, se apoya una estructura cuadrada de esquinas redondeadas similar a los edificios típicos de este período (Fig. 4:4) (Yaroshevich, 2016: Fig. 22).

En la cima de Givat Rabi, a gran altura sobre el área del asentamiento donde se llevó a cabo la excavación, los autores examinaron numerosas acumulaciones y restos del período Bronce Antiguo IB y el período Bronce Antiguo II. Al oeste de la cúpula, en el borde del antiguo asentamiento, hay un amplio terraplén y es probable que represente los restos de un muro. También es probable que las dos secciones sean partes del mismo muro que lo rodeaba: un área al pie de la pendiente cerca de Ein Zippori, una zona rocosa en el centro de la pendiente y un asentamiento superior en la cima de la colina. El área total de la ciudad que rodeaba esta muralla estimada era de unas 50 hectáreas (Fig. 2). 

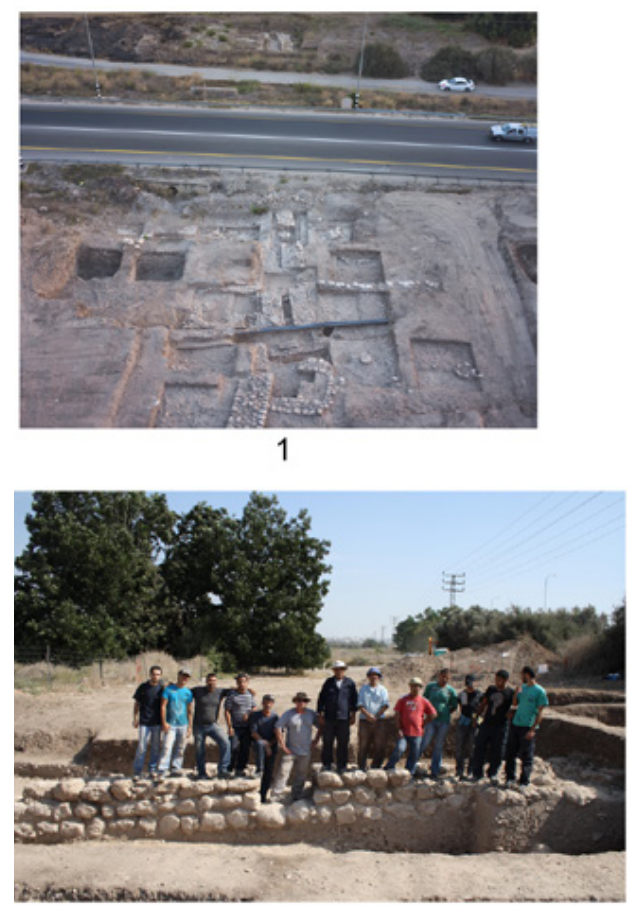

3

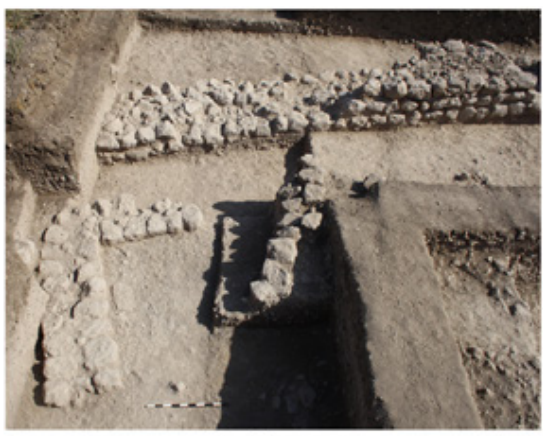

2

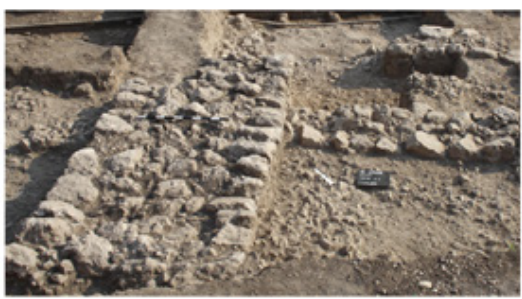

4

Fig. 4. Ein Zippori, fortificación de las áreas D3 y N5. 1) Vista general hacia el norte (cortesía Ala Yaroshevich, IAA). 2) Casa cerca de la fortificación en el Area D3, vista hacia el este (foto Nimrod Getzov, IAA). 3) Enno Brun, supervisor de área, y los obreros sobre la fortificación en el Area D3, vista hacia el este (foto Nimrod Getzov, IAA). 4) Vista hacia el nordeste de la torre adjunta a la fortificación en el Area $\mathrm{N}_{5}$ (cortesía de Ala Yarosevich, IAA).

Entre las vasijas de alfarería encontradas en este estrato, se destacaron tipos comunes en los conjuntos cerámicos de la cuenca del mar de Galilea, tales como: cuencos con revestimiento agrietado (Crackled Ware, Esse, 1989) y fosas con borde engrosado cortado en círculo con decoración de cono. Gran parte de los recipientes de almacenamiento están decorados con la decoración de bandas llamada Grain Wash Ware (Amiran, 1969: foto 42; Braun, 1985: Fig. 19:10-1). También se encontraron dos improntas de sellos con patrones geométricos típicos del Bronce Antiguo II, pero sobre cerámica típica del Bronce Antiguo IB (Paz et al., 2018).

\section{Edad del Bronce Antiguo II}

De la Edad del Bronce Antiguo II (principios del III milenio a.C.) se hallaron vasijas de cerámica, principalmente platos y tinajas de la familia de vasijas metálicas, la mayoría en áreas ubicadas sobre los restos del Bronce Antiguo IB. En estos contextos se encontraron pocos restos de edificios, con esquinas en ángulo recto que diferían de las esquinas redondeadas de las construcciones de estrato anteriores. En un relevamiento en la parte superior de Givat Rabi se pudo constatar también la presencia de muchos fragmentos de cerámica de este período (Fig. 2). 


\section{Las lámparas de aceite de Ein Zippori}

Se recogió todo el suelo excavado durante la excavación, tamizando 21.523 tiestos de vasijas cerámicas del período Bronce Antiguo IB, 303 de ellos pertenecientes a lámparas de aceite (Figs. 5, 6). Hay que tener en cuenta que en los aproximadamente mil bordes de cuencos del período Calcolítico temprano encontrados en el sitio, no se identificó ninguno con marcas de quemaduras u hollín.

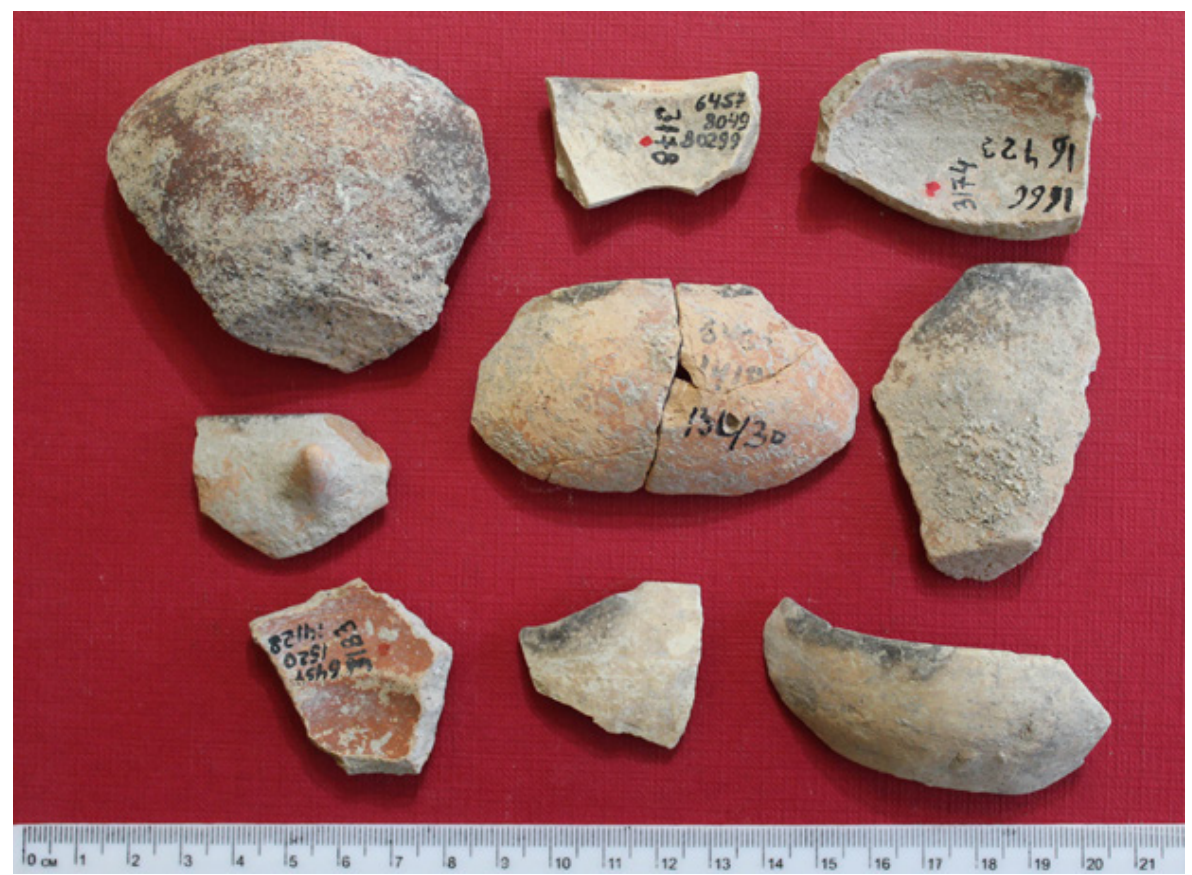

Fig. 5. Foto de diferentes lámparas de aceite de Ein Zippori (foto Nimrod Getzov, IAA).

Un análisis de materiales orgánicos residuales en vasijas de cerámica de Ein Zippori mostró que el uso de aceite de oliva en el sitio comenzó a ser empleado al final del período Neolítico Cerámico en los estratos que atribuimos al llamado "Horizonte Nahal Zippori". También se tomaron muestras de utensilios del Bronce Antiguo IB, incluidos cuencos, y se encontró que muchos de ellos se usaban para almacenar aceite de oliva (Namdar et al., 2015). 

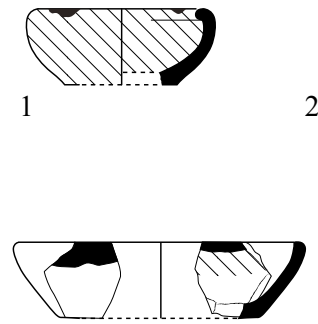

5

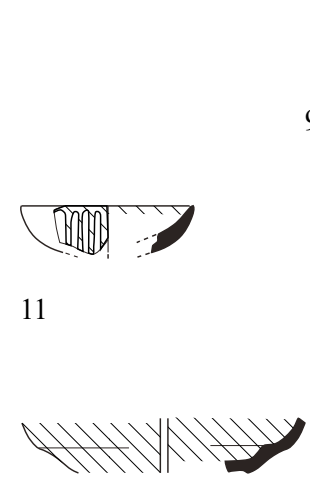

15

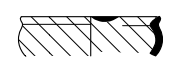

18

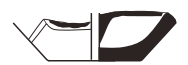

21

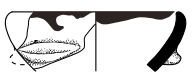

9

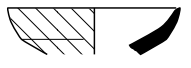

12

16
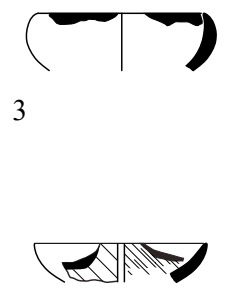

7

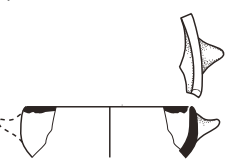

10
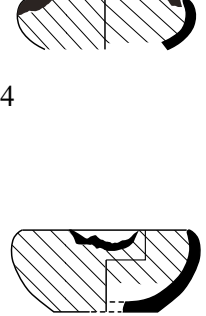

8

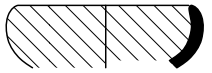

13

14
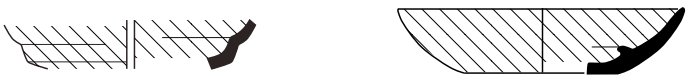

17

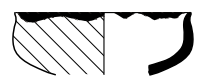

19

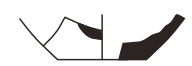

22

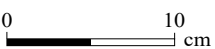

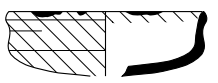

20

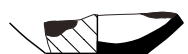

23

Fig. 6. Dibujos de varias lámparas de aceite de Ein Zippori (dibujos Hagit Tarhan, IAA).

Las lámparas de aceite que se encuentran en Ein Zippori pueden dividirse en estos cuatro grupos:

1) Cuencos con un borde simple que generalmente tiene un borde puntiagudo (Fig. 6: 1-14). En la mayoría de los casos, se encontraron marcas de quemaduras en los bordes de los tazones y, en algunos casos, una pequeña abolladura se rompe en el borde para colocar la mecha y luego no hay marcas de quemaduras en otros lugares (Fig. 6: 6-8). Se puede suponer que los fragmentos en los que no hay marcas de quemaduras pertenecen a tales cuencos. Y todos los cuencos pequeños con el borde simple fueron creados para ser usados como lámparas.

2) Lámparas graduadas que incluían un pequeño estante que evitaba que la mecha se deslizara hacia el fondo del recipiente (Figs. 6: 15-17, 7:1). No 
se encontraron lámparas de este tipo en la excavación actual en la que sobrevivió un perfil completo, desde el borde hasta la base, pero en una excavación previa realizada por Barzilai en el sitio, se encontró uno de esos elementos (Fig.6: 17, Barzilai et al., 2014: Fig. 9: 7).

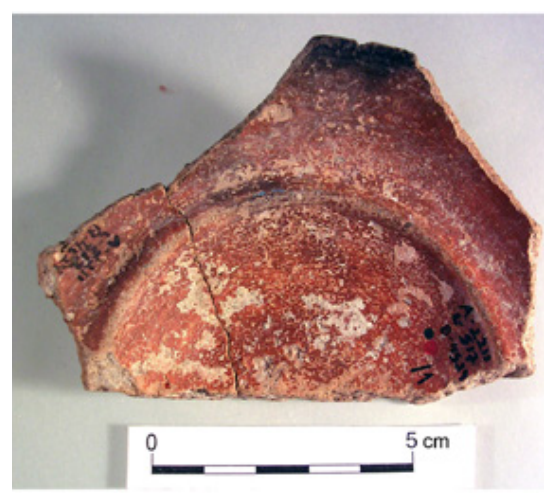

1

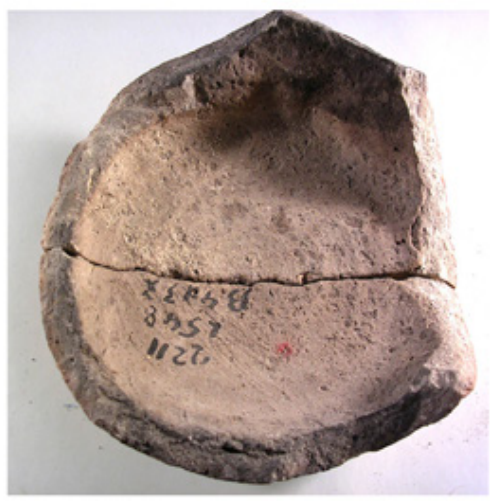

3

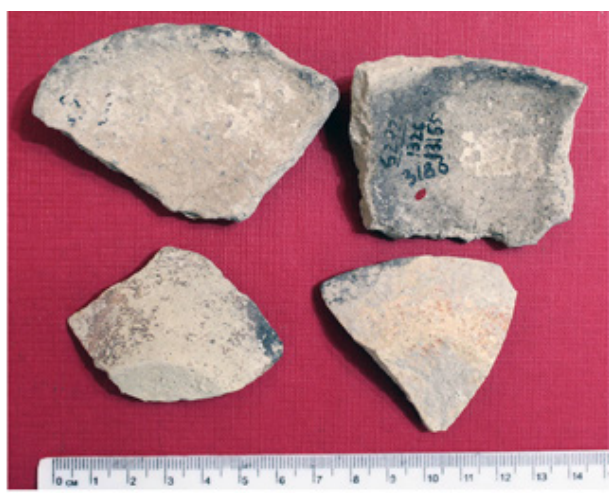

2

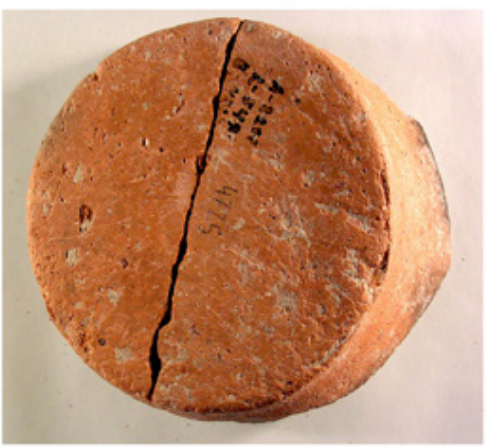

4

Fig. 7. Bases de diferentes lámparas de aceite de Ein Zippori (foto Nimrod Getzov, IAA).

3) Cuencos con la cabeza del borde hacia afuera y formando una especie de estante estrecho (Fig. 6: 18-20). Sólo se encontraron unos pocos cuencos de este tipo en la excavación y la mayoría de ellos mostraba marcas de quemaduras en el borde. Por un lado, se puede suponer que todos fueron hechos para uso de lámparas. Por otro lado, debido a su rareza en Ein Zippori, también es posible que éste sea un uso aleatorio de cuencos originarios de otras áreas y al llegar aquí desconocemos su propósito original.

4) Bases de varias vasijas que fueron rotas y reutilizadas como lámparas de aceite, aquí también el quemado del extremo roto de la vasija indica su uso como lámpara (Fig. 6: 21-23, Fig. 7:2). 


\section{Lámparas de aceite en varios conjuntos cerámicos protohistóricos}

Se han encontrado lámparas de aceite antiguas en muchos otros sitios; para conocer su distribución notaremos hallazgos de lámparas o su ausencia en algunos complejos protohistóricos de la región. Es importante señalar que muchos informes de excavaciones no indicaron expresamente en el texto el uso de lámparas de aceite y no indicaron marcas de quemaduras en los cuencos. La evidencia de estos signos a menudo se puede encontrar en las tablas de descripción de las vasijas de cerámica. Con el fin de garantizar información directa sobre las marcas de quemaduras en los cuencos, en la lista de los sitios que se describen a continuación se hizo hincapié en las excavaciones en las que participaron los autores. No hace falta decir que la lista a continuación incluye sólo sitios seleccionados y las lámparas de aceite de la Edad del Bronce Antiguo también aparecen en muchas otras publicaciones (por ej., Marquet-Krause, 1949; Douglas y Kafafi, 2000; Kaptijn, 2009; Bar et al., 2012).

Ard el-Samra: extenso sitio protohistórico que cubre un área de aproximadamente 20 hectáreas (Getzov et al., en prensa). Cuenta con restos del período Calcolítico temprano y tardío, pero entre los cuales no se encontró evidencia del uso de lámparas de aceite. Los primeros cuencos que se utilizaron como lámparas de aceite se hallaron en un estrato del Bronce Antiguo IB. A pesar del gran tamaño del sitio, no hay datos suficientes para determinar el área del asentamiento en cada uno de los períodos.

Qiryat Ata: asentamiento rural del Bronce Antiguo IB en los estratos II-III, cubriendo un área de aproximadamente 2,5 hectáreas, y una pequeña ciudad que cubre un área de aproximadamente 3,5 hectáreas en el Estrato I (Golani, 2003). Muchos de los cuencos pequeños sirvieron como lámparas en el Estrato II (Golani, 2003: 88) y también se encontró un recipiente con hollín en el Estrato I del Bronce Antiguo II (Golani, 2014: Fig. 13:4).

Tel Qashish: asentamiento de más de 4 hectáreas (Ben Tor et al., 2003: 1; van den Brink, 2014), en cuyos estratos XV-XIII se encontraron restos del Bronce Antiguo IB (Ben Tor et al., 2003: Fig. 9:1).

Megiddo: yacimiento que data del Neolítico y en el Bronce Antiguo IB se convirtió en un gran asentamiento con un templo inusual (Ussishkin, 2014). En la ladera sureste conocida como Megiddo Stages (Engberg y Shipton, 1934; Braun, 2013) se hallaron pequeños cuencos de las etapas VII-IV (Bronce Antiguo IB) con hollín, por lo que Charloux los definió como lámparas (Charloux, 2006: tabla 36:11,10). En las re-excavaciones en el tell, en el Estrato J6, que data del Bronce Antiguo III, se encontró una gran cantidad de cuencos pequeños con hollín en los bordes que según los autores sirvieron como lámparas (Greenberg et al., 2006: 162, Figs. 10.4:1, 10.5: 1,9,11). También se encontraron muestras de hollín en los bordes de cuencos del Estrato J7, que es el relleno de los cimientos del Templo 4040, también del Bronce Antiguo III (Adams, 2013: Fig. 8.17: 7).

En Shadud: sitio extenso del Bronce Antiguo IB, con un área de al menos una decena de hectáreas. En esta etapa del estudio no se sabe si el asentamiento 
estaba rodeado por un muro. Se encontraron cuencos con marcas de quemaduras (Braun, 1985: Fig. 18:4,2) y dos lámparas escalonadas (Braun, 1985: Fig. 18:6-7).

Tel Bet Yerah: extenso montículo que sirvió como asiento de ciudades con un área de más de 30 hectáreas con muchas expediciones a lo largo de décadas (por ej., Getzov, 2006; Greenberg et al., 2006). Se excavó una parte de un pequeño asentamiento del Bronce Antiguo IA, y, entre otras cosas, se encontraron cuencos pequeños y simples que servían como lámparas de aceite (Greenberg et al., 2013: Figs. 13:4-3, 18:8). Durante el período del Bronce Antiguo IB, los autores de este artículo creen que había una ciudad grande y fortificada (Getzov, 2006: cap. 2). En la excavación 1994-5 se encontraron lámparas, principalmente cuencos simples (Getzov, 2006: Fig. 2.12:25-29) y dos lámparas escalonadas (Fig. 7:3) (Getzov, 2006: Fig. 2.12:30-31). También se encontraron lámparas de aceite en las otras áreas de excavación en restos datados del Bronce Antiguo IB (por ej., Eisenberg y Greenberg, 2006: Fig. 47.8:1). Durante el Bronce Antiguo II, el uso de lámparas de aceite en Bet Yerah también era común, pero es interesante que en ese momento se preferían los cuencos poco profundos (Getzov, 2006: Fig. 41.3:4-3; Eisenberg y Greenberg, 2006: Fig. 65.8:1-2) y también se usaban bases recicladas (Fig.7: 4) (Getzov, 2006: Fig. 44.3:18; Eisenberg y Greenberg, 2006: Fig. 59.8:1).

Tel Yaqush: sitio que tiene un tamaño de 2-3 hectáreas cuyos restos se extienden desde el período de Bronce Antiguo IA al Bronce Antiguo III. La mayoría de los hallazgos del sitio pertenecen a Bronce Antiguo IB (Rotem, 2015: 205-146). Los cuencos pequeños constituyen el uno por ciento del complejo y, según Rotem: "Incluidos los pequeños cuencos tienen marcas de hollín en el borde, y parecen haber sido utilizados como lámparas” (Rotem, 2015: 190).

Tel Bet Shean: asentamiento de unas 4 hectáreas (Rotem, 2015: 232). Durante el Bronce Antiguo IB los cuencos pequeños eran comunes y la mayoría de ellos se usaban como lámparas (Rotem, 2012: 134). Probablemente destinados a tender un hilo (Rotem, 2012: 137, foto 4.7).

Tel Shalem: asentamiento fortificado con una amplia muralla del Bronce Antiguo IB, que cubre un área de aproximadamente 5 hectáreas. Las marcas de hollín en algunos de los cuencos pequeños indican que fueron utilizados como lámparas (Eisenberg, 1996: 9). Parece que en uno de los cuencos pequeños (Eisenberg, 1996: Fig. 13:3) se rompió una pequeña cavidad en el borde para colocar la mecha.

Mishmar Haemeq: sitio que cubre varias hectáreas en el período Neolítico precerámico, ocupado también durante el período Calcolítico y el Bronce Antiguo I (Getzov y Barzilai, 2011). En el Bronce Antiguo IA no se encontró evidencia del uso de lámparas de aceite, pero sí en pequeñas cantidades en el Bronce Antiguo IB.

Ein Asawir: sitio que contiene restos de asentamientos protohistóricos incluyendo el Bronce Antiguo IB en el que el sitio cubría un área de aproximadamente 60 hectáreas (Elad et al., 2020). Tumbas del Bronce Antiguo IB fueron 
expuestas al este del sitio, donde también se encontraron cuencos que se usaban como lámparas (Yannai, 2016: 48, tipo BB).

Tel Aphek: sitio que consistía en una ciudad fortificada con un área de 12 hectáreas en la Edad del Bronce Antiguo IB, donde también se encontraron los restos de un asentamiento del Bronce Antiguo II y períodos posteriores. Aparecen marcas de hollín en uno de los platos típicos de la despensa para el paso del Bronce Antiguo IB al II (Beck, 1985: Fig. 4:11; Milevski, 2016: 98, Fig. 3.13:18).

Tel Dalit: restos de un asentamiento rural del Bronce Antiguo IB y de un asentamiento rodeado por un muro que cubre un área de unas 4 hectáreas del Bronce Antiguo II. Un cuenco carenado datado del paso del Bronce Antiguo IB al II muestra restos de hollín (Gophna, 1996: Fig. 39:13).

Nesher-Ramle: un cementerio que cubre unas 12 hectáreas con cuevas del período Calcolítico tardío, Bronce Antiguo IB y períodos posteriores. La cueva F-565 tiene varios cuencos con marcas de hollín en el borde (Avrutis, 2012: tabla $4.7: 32$, 41; 4.8: 14). En la cueva F-355 se encontraron jarras con marcas de hollín en los costados (Avrutis, 2012: tabla 4.2: 8, 10).

Tel Yarmuth: un sitio con un área de aproximadamente 16 hectáreas. En el lugar se han encontrado restos arqueológicos desde la Edad del Bronce Antiguo IB hasta el Bronce Antiguo III, aunque la mayoría de los hallazgos del sitio pertenecen al Bronce Antiguo III (de Miroschedji, 1988). Un estudio de cuencos pequeños del Área B, Estrato B-V (Bronce Antiguo IB) fue definido por Charloux (2006: 44, tabla 22:20-30) como lámparas porque tenían marcas de hollín en el borde. También hay pequeños cuencos con hollín en las áreas B y C, Estrato B-II y C-IV, que pertenecen al Bronce Antiguo III (de Miroschedji, 1988: tablas 28:1, 32: 6).

Beqoa: sitio que tiene un tamaño de aproximadamente 9 hectáreas. La mayoría de los hallazgos pertenecen al período Calcolítico tardío y al Bronce Antiguo IB y II (Golani et al., 2018). En las excavaciones renovadas por N. Ben-Ari (información oral) también se han encontrado restos del Bronce Antiguo IA. El director de la excavación definió como lámparas de aceite un pequeño número de tazones pequeños que datan del Bronce Antiguo IB porque tenían marcas de hollín en el borde.

Tel Erani: sitio que mide 25 hectáreas con los restos de un asentamiento cuya mayoría de hallazgos pertenecen a una ciudad fortificada de la Edad del Bronce Antiguo IB1 y otras construcciones del Bronce Antiguo IB2, II y III, identificadas en una serie de excavaciones diferentes en el yacimiento (Yeivin, 1961; Kempinski y Gilead, 1991; Ciałowicz et al., 2016; Milevski et al., 2016; 2019). Los cuencos que se utilizaron para lámparas se encontraron en los estratos del Bronce Antiguo IB1 en el Área Q, como evidencia de marcas de hollín en los bordes de cuencos cerámicos; aparentemente se encuentran otros casos como estos en otras áreas.

Arad: sitio que mide 9 hectáreas y existió desde el período Calcolítico tardío hasta el Bronce Antiguo II. La mayoría de los hallazgos del sitio pertenecen 
al asentamiento del Bronce Antiguo IB y una ciudad fortificada del período Bronce Antiguo II (Amiran, 1978; Amiran e Ilan, 1996). Tazones pequeños de los Estratos II-III (Bronce Antiguo II) fueron definidos por Amiran (1978: 44, tabla 22:20-30) como lámparas de aceite porque tenían marcas de hollín en los bordes.

Tell Iktanu: sitio que contiene restos del Bronce Antiguo y del Bronce Intermedio (Prag, 1991; 2000). Los restos del período del Bronce Antiguo IB cubren unas 3 hectáreas. Prag (2000: 98) menciona pequeños cuencos semiesféricos con bordes llenos de hollín que deben haber sido utilizados como lámparas de aceite.

Bab edh-Dhra: asentamiento de unas 4 hectáreas, habitado desde el período del Bronce Antiguo IA hasta la Edad del Bronce Intermedio. Sólo uno de los 120 cuencos del asentamiento que se han publicado pertenecientes al Bronce Antiguo IB-II tiene marcas de hollín (Schaub, 2000: 454-455).

\section{Discusión: Entre la urbanización, la división del trabajo y el uso de lámparas}

Los cuencos utilizados como lámparas de aceite primitivas, en los primeros asentamientos de la Edad del Bronce, son conocidos en la investigación y mencionados por muchos estudiosos (por ej., Kaplan, 1952; Sussman, 1985; Charloux, 2006), pero no recibieron un estudio cronológico y tipológico detallado. Ben-Ari también señaló el uso de cuencos de iluminación dentro de las cuevas funerarias de Bronce Antiguo IB en la llanura costera, donde se han encontrado también varias lámparas (Ben-Ari, 2010: 82).

Los hallazgos nos permiten definir, a grandes rasgos, cuatro grupos diferentes de lámparas según la tipología de las vasijas. La mayoría (grupos 1,2) eran, en nuestra opinión, cuencos diseñados específicamente con esta finalidad. Las bases que fueron recicladas y convertidas en lámparas de aceite (grupo 4) probablemente fueran de uso ocasional. Sólo en unos pocos casos no está claro si fueron fabricados como lámparas o si su uso fue ocasional (grupo 3). Un tipo especial que no se encuentra en Ein Zippori son los tipos que se identifican en Beit Shean, los cuales parecen haber tenido una función ceremonial y no eran de uso diario.

En lo que respecta a las inmediaciones de Ein Zippori, no contamos con evidencias del uso previo de lámparas. Por ejemplo, en Einot Kaukab, un sitio del Calcolítico tardío que excavamos, no se encontraron marcas de hollín en el borde de los cuencos (Getzov, 2016). Tampoco se encontraron lámparas en los estratos del Bronce Antiguo IA en Yiftahel, otro sitio cercano a Ein Zippori (Braun, 1997). El único lugar del Bronce Antiguo IA donde se han encontrado lámparas es Tel Bet Yerah. Por lo tanto, parece que el cambio hacia el uso diario ocurrió recién en el Bronce Antiguo IB.

A partir de entonces, la distribución de lámparas de aceite en complejos antiguos no cambió sustancialmente y, a modo de ilustración, hemos recopilado datos 
de cuatro complejos seleccionados de diferentes períodos (Tabla 2). El complejo del Bronce Antiguo IB en el sitio de Ein Zippori, discutido anteriormente; un complejo de la Edad del Bronce Tardío (ca. 1400 a.C.) identificado en Nahariya (Amitzur et al., 2016; según los datos facilitados por Ron Beeri); un complejo del período bizantino en Horbat Ovesh (Avshalom Gorny, 1998); y un complejo del período mameluco ubicado en el distrito El Vuta de Safed (Dalali-Amos y Getzov, 2019). Los datos del período bizantino en Horbat Ovesh son más altos y dependen del hecho de que en esa época las lámparas se hacían en moldes y los cálculos por unidad son diferentes. De todos modos, el análisis de datos de otras épocas sobre lámparas de aceite excede los fines de este artículo.

\begin{tabular}{ccccc}
\hline Períodos & $\begin{array}{c}\text { Bronce } \\
\text { Antiguo IB }\end{array}$ & $\begin{array}{c}\text { Bronce } \\
\text { Tardío }\end{array}$ & Bizantino & Mameluco \\
\hline Sitios & Ein Zippori & Nahariya & $\begin{array}{c}\text { Horbat } \\
\text { Ovesh }\end{array}$ & Safed \\
\hline $\begin{array}{c}\text { Total de bordes en el } \\
\text { conjunto cerámico }\end{array}$ & 21.532 & 6.717 & 3.044 & 2.567 \\
\hline $\begin{array}{c}\text { Total de fragmentos } \\
\text { de lámparas de } \\
\text { aceite }\end{array}$ & 303 & 125 & 125 & 50 \\
\hline $\begin{array}{c}\text { Porcentaje de las } \\
\text { lámparas dentro del } \\
\text { conjunto cerámico }\end{array}$ & $1,4 \%$ & $1,9 \%$ & $4,1 \%$ & $1,9 \%$ \\
\hline
\end{tabular}

Tabla 2. Distribución de lámparas en sitios seleccionados de diferentes períodos.

Durante la Edad del Bronce Antiguo II y III, los sitios y la población del Levante meridional se expandieron, se construyeron palacios o proto-palacios (de Miroschedji, 2012-2013) y se establecieron las primeras ciudades fortificadas (Milevski et al., en prensa). Artesanos, comerciantes y servidores del gobierno y otros sectores de las clases bajas, principalmente campesinos, soportaron la carga de la producción de alimentos y las necesidades de todas las clases, incluidos los artículos de lujo para las clases altas (Milevski, 2016: 240-243).

Después del período Calcolítico, a principios de la Edad del Bronce, la distribución de bienes a través del trueque se expandió y de ella resultó una mayor división del trabajo (Charloux, 2006; Milevski, 2011; 2013; 2016). Esta división del trabajo consistió en la especialización en más artesanías y un número creciente de personas dedicadas a trabajos únicos y diferentes (Costin, 1991; 2020).

Según economistas clásicos como Adam Smith (1904 [1776]: 3) y Karl Marx (1970 [1859]: 52-51) esta división del trabajo produce un efecto de doble producción, constituyendo una división entre trabajadores manuales e intelectuales, entre explotadores y explotados, afectando también la división entre la ciudad o localidad urbana y el área rural circundante. Smith vio esto como una señal positiva, mientras que Marx consideró este progreso como una contradicción porque los productores físicos habían perdido el control de los medios de producción, como tierras, talleres, etc. De manera similar, Gordon Childe (1950) vio el proceso que llamó la "revolución urbana" como un componente negativo (Lamberg-Karlovsky, 2009). 
Uno de los cambios que se produjo al inicio del Bronce Antiguo I fue el aumento de la producción y la distribución de productos a mayores distancias en el Levante meridional (Milevski, 2016). Una de las cosas que influyó en esto fue la domesticación del asno y la posibilidad de viajes más largos (Milevski y Horwitz, 2019).

La expansión de la especialización en los asentamientos rurales y especialmente en los asentamientos urbanos del Bronce Antiguo IB probablemente propició la ampliación de la jornada laboral, por lo que fue necesario utilizar lámparas de aceite, para la iluminación de los talleres, durante las horas de oscuridad como las descritas en este artículo.

Es posible que la expansión del uso de las cuevas funerarias en el Bronce Antiguo IB (Ben-Ari, 2010; Milevski, 2019) también condujera al uso de lámparas para iluminar el interior de aquellas, pero también es posible que estas lámparas fueran pertenencias personales de los enterrados con ellas, así como otros utensilios de cerámica y ajuar diverso. Y es posible que el uso de lámparas en los templos se haya realizado no sólo por la luz sino por los componentes de la "magia" que en la antigüedad se asociaban con el uso del fuego (por ej., Gošić y Gilead, 2015).

\section{Conclusión}

A partir de los datos aquí presentados, parece que el uso generalizado de lámparas de aceite aparece durante la Edad del Bronce Antiguo IB en todo el Levante meridional: en pequeños asentamientos rurales, grandes asentamientos y ciudades recién fundadas. Sugerimos que al extender la carga de trabajo a las clases bajas (campesinos, artesanos, servidores) debe haber extendido la jornada laboral a las horas de la oscuridad. Esto incluyó a manualidades que se pueden hacer en el hogar.

Si se acepta esta explicación, entonces los hallazgos arqueológicos de las lámparas pueden verse como un eco de los profundos cambios sociales que impulsó la revolución urbana. Los hallazgos de las lámparas también indican que los cambios abarcaron a toda la población, ya en el Bronce Antiguo IB, desde los habitantes de las aldeas hasta los de las ciudades recién fundadas.

Moses Finley (1987-1989), al escribir sobre la ciudad en los períodos clásicos de la antigüedad, dijo que es muy difícil captar el "sentimiento" de una ciudad antigua; nosotros agregamos que esta dificultad es mucho mayor en el caso de un asentamiento de la Edad del Bronce Antiguo. Sin embargo, podemos imaginar la experiencia de los habitantes de esos asentamientos, con luz y lámparas, en actividades en las horas de oscuridad, lo cual puede acercarnos a este sentimiento por las razones que hemos señalado anteriormente.

Al hablar de la revolución urbana, Gordon Childe (1950: 3) tenía en mente la revolución industrial en Inglaterra. La misma significó un cambio en la organización de las comunidades que provocó y fue acompañado de un cambio 
dramático en la sociedad y la población. Las dos últimas de sus diez características de la revolución urbana en la Edad del Bronce, fueron la existencia de un plusproducto social, un grupo de artesanos especialistas y una organización basada más en la residencia que en el parentesco (Childe, 1950: 16). Es interesante que, al investigar la revolución industrial desde un punto de vista arqueológico, West (2017) destacó que la evolución de las fábricas, y entre ellas, las de finales del siglo XVIII, jugó un papel vital en el desarrollo de la iluminación artificial, particularmente en el uso de luces de gas. Esta iluminación artificial fue una de las características que permitió también avances en la producción durante toda la jornada laboral.

Destacamos el significado de nuestros hallazgos para la iluminación artificial de una sociedad que hace más de 5000 años pasó de una organización protourbana a una urbana. Esto significa que las lámparas de aceite participaron en la reorganización de esas comunidades de la Edad del Bronce en lo que respecta no sólo a una jornada de trabajo más larga, sino también a la forma de organizar los trabajos en torno a las luces. Antes, los trabajos se realizaban durante el día, utilizando la luz natural, o alrededor de hogueras o antorchas (Pettitt et al., 2017).

Aunque la domesticación del fuego ya se hizo en el Levante y otras partes del mundo durante el período Paleolítico (Alperson-Afil, 2008; Berna et al., 2012), y los períodos Neolítico y Calcolítico dominaron la pirotecnología para la producción de artefactos de empasto, arcilla y cobre (Goren, 1991; Levy, 2007; Goren y Goring-Morris, 2008), la Edad del Bronce Antiguo y su fase urbana domesticaron la luz, "inventando" la luz artificial en forma de lámparas de aceite.

La utilización de tales lámparas de aceite podría proporcionar una mejor iluminación para tareas que normalmente requieren luz solar directa como decoración de cerámica y figurillas, tallado de pedernal, y muchas otras tareas que en el pasado se realizaban sólo durante el día. Puede ser que incluso determinadas áreas en los centros urbanos y otros asentamientos se hayan dividido en actividades de luz diurna y nocturna, incluidas actividades no económicas (Papadopoulos et al., 2015). La domesticación de olivos y la producción de aceite, y un sistema de intercambio de olivos durante la Edad del Bronce Antiguo (Milevski, 2011, con bibliografía), por supuesto, deberían haber contribuido a la existencia de esas lámparas teniendo en cuenta a la preexistente fabricación de cerámica. Debemos recordar que varias vasijas de cerámica de Ein Zippori contenían aceite de oliva (Namdar et al., 2015), aun así, era posible utilizar otras grasas y lípidos para el combustible (Papadopoulos et al., 2015).

Las lámparas de aceite más portables, más manejables y que ahorraban más energía deben agregarse a la lista de las diez características de Childe sobre la revolución urbana, al menos en el sur de Levante. Además, las percepciones sensoriales que brinda la luz de las lámparas y las prácticas realizadas con su luz interactúan en estas comunidades, no sólo en los espacios urbanos, sino también en las aldeas y las cuevas funerarias que configuran las nuevas percepciones y sentimientos, que por supuesto todavía nos resultan difíciles de entender. 


\section{Agradecimientos}

Este artículo tiene como antecedentes una charla presentada por Nimrod Getzov en 2019 en la Academia Kinneret al borde del Mar de Galilea, y un artículo en hebreo presentado por los autores para un volumen a publicarse en Jerusalén. Queremos agradecer a Pablo Jaruf por la dedicada edición de este artículo en castellano y sus sugerencias al respecto que han permitido obtener un texto más claro. Asimismo, queremos agradecer al departamento de Publicaciones de la Autoridad de Antigüedades por el permiso de publicar material inédito. 


\section{Q Bibliografía}

》Adams, M. J. (2013). The Early Bronze Age Pottery from Area J, en: Finkelstein, I., Ussishkin, D. y Cline, E. H. (eds.), Megiddo V. The 2004-2008 Seasons Volume II. Tel Aviv: Tel Aviv University, 295-334.

»Alperson-Afil, N. (2008). Continual Fire-Making by Hominins at Gesher Benot Ya'aqov, Israel, en: Quaternary Science Reviews 27: 1733-1739.

"Amiran, R. (1969). The Ancient Pottery of the Holy Land. From its Beginning in the Neolithic Period to the Iron Age. Jerusalén: Israel Exploration Society.

"Amiran, R. (1978). Early Arad I. The Chalcolithic and Early Bronze City, First-Fifth Seasons of Excavations, 1962-1966. Jerusalén: Israel Exploration Society.

» Amiran, R. y Ilan, O. (1996). Early Arad II. The Chalcolithic and Early Bronze IB Settlements and the Early Bronze II City: Architecture and Town Planning, Sixth to Eighteenth Seasons of Excavations, 1971-1978, 1980-1984. Jerusalén: Israel Exploration Society.

» Amitzur, Y., Be`eri, R. y Getzov, N. (2016). Tel Nahariyya, en: Hadashot Arkheologiyot - Excavations and Surveys in Israel 128. https://www.hadashot-esi. org.il/report_detail_eng.aspx?id=25093\&mag_id=124 (accedido 7/3/2021)

»Avrutis, V. W. (2012). Late Chalcolithic and Early Bronze Age I Remains at NesherRamla Quarry. Haifa: University of Haifa.

»Avshalom-Gorny, D. (1998). Horvat Obesh, en: Hadashot Arkheologiyot Excavations and Surveys in Israel 108: 68 (hebreo).

" Bar, S., Cohen, O., Zuckerman, R. y Winter, H. (2012). The Archaeological Excavations at the Early Bronze Age I site Fazael 4, Jordan Valley, Israel, en: Mitekufat Haeven - Journal of the Israel Prehistoric Society 42: 193-226.

»Barzilai., O., Getzov, N., Gobenko, N., Marom, N., Milevski, I., Vered, A. yZheng, J. F. (2014). Proto-Historic Ein Zippori: The 2007 Excavation Season, en: Mitekufat Haeven - Journal of the Israel Prehistoric Society 37: 22-72.

»Beck, P. (1985). An Early Bronze Age Family of Bowls from Tel Aphek, en: Tel Aviv 12: $17-28$.

»Ben-Ari, N. (2010). Costumbres funerarias en la región costera de Israel en la Edad del Bronce Antiguo IB y su contribución a la comprensión de la sociedad protourbana. Tesis de maestría. Tel Aviv: Universidad de Tel Aviv (hebreo).

»Ben-Tor, A., Bonfil, R. y Zuckerman, S. (2003). Tel Qashish, a Village in the Jezreel Valley, Final Report of the Archaeological Excavations. Jerusalén: The Hebrew University of Jerusalem.

» Berna, F., Goldberg, P., Horwitz, L. K., Brink, J., Holt, S., Bamford, M. y Chazan, M. (2012). Microstratigraphic evidence of in situ fire in the Acheulean strata of Wonderwerk Cave, Northern Cape province, South Africa, en: Proceedings of the National Academy of Sciences 109 (20): E1215-E1220; https://doi.org/10.1073/ pnas.1117620109 (accedido 7/9/2021).

"Braun, E. (1985). En Shadud, Salvage Excavations at a Farming Community in the Jezreel Valley, Israel. Oxford: BAR. 
»Braun, E. (1997). Yiftah 'el. Salvage and Rescue Excavations at a Prehstoric Village of the Lowere Galilee, Israel (IAA Reports 2). Jerusalén: Israel Antiquities Authority.

»Braun, E. (2013). Early Megiddo on the East Slope (The "Megiddo Stages"), A Report on the Early Occupation of the East Slope of Megiddo, Results of the Oriental Institute's Excavations, 1925-1933. Chicago: The Oriental Institute of the University of Chicago.

" van den Brink, E. C. M. (2014). Tel Qashish (Rakevet Haemeq), en: Hadashot Arkheologiyot - Excavations and Surveys in Israel 126. https://www.hadashot-esi. org.il/report_detail_eng.aspx?id=14714\&mag_id=121 (accedido 5/3/2021)

»Charloux, G. (2006). Artisanat et urbanization de la Palestine à l'âge du Bronze Ancien. Aport de l'étude des céramiques à la structure sociale. Tesis de doctorado. París: Université Pantheon-Sorbonne.

»Childe, V. G. (1950). The Urban Revolution, en: The Town Planning Review 21 (1): 3-17.

»Ciałowicz, K., Yekutieli, Y. y Czarnowicz, M. (eds.) (2016). Tel Erani I. Preliminary Report of the 2013-2015 Excavations. Cracovia: Wydawnictwo Alter.

»Costin, C. L. (1991). Craft Specialization: Issues in Defining, Documenting, and Explaining the Organization of Production, en: Schiffer, M. B. (ed.), Archaeological Method and Theory 1. Tucson: Academic Press, 1-56.

" Costin, C. L. (2020). What is a Workshop?, en: Hodgkinson, A. K. y Lelek Tvetmarken, C. (eds.), Approaches to the Analysis of Production Activity at Archaeological Sites. Oxford: Oxbow Books, 177-197.

»Dalali-Amos, E. y Getzov, N. (2019). Remains from the Mamluk Period in the alWatța Quarter, Safed (Zefat) (with a contribution by Oded Katz), en: 'Atiqot 97: $1^{*}-95^{*} ; 271-275$ (hebreo con resumen en inglés).

"Douglas, K. y Kafafi, Z. (2000). The Main Aspects of the Early Bronze I Pottery from Jebel Abu Thawwab. North Jordan, en: Philip, G. y Baird, D. (eds.), Ceramics and Change in the Early Bronze Age of the Southern Levant. Sheffield: Sheffield Academic Press, 279-286.

»Elad, I., Paz, Y. y Shalem, D. (2020). 'En Esur (Asawir) Areas K y L, en: Hadashot Arkheologiyot -Excavations and Surveys in Israel 132. https://www.hadashot-esi. org.il/report_detail_eng.aspx?id=25724\&mag_id=128 (accedido 20/5/2020).

»Eisenberg, E. (1996). Tel Shalem, Soundings in a Fortified Site of the Early Bronze Age IB, en: 'Atiqot 30: 1-24.

»Eisenberg, E. y Greenberg, R. (2006). Area EY: The Eisenberg-Yogev Excavation, 1981-1982, 1985-1986, en: Greenberg, R., Eisenberg, E., Paz, S. y Paz, Y., Beth Yerah, The Early Bronze Age Mound. Vol. I, Excavations Reports, 1933-1986. Jerusalén: Israel Antiquities Authority, 339-468.

»Engberg, R. M. y Shipton, G. M. (1934). Notes on the Chalcolithic and Early Bronze Age Pottery of Megiddo. Chicago: The Oriental Institute of the University of Chicago.

»Esse, D. L. (1989). Village Potters in Early Bronze Age Palestine: a Case Study, en: Leonard, A. Jr. y Williams, B. B. (eds.), Essays in Ancient Civilization Presented to Helene J. Kantor. Chicago: The Oriental Institute of the University of Chicago, 77-92.

» Finley, M. (1987-1989). The City, en: Opus 6-8: 305-315. 
"Gal, Z. (2002). Settlement Location in Nahal Zippori as a Reflection of Cultural Diversity from the Neolithic through the Early Bronze Age II-III periods, en: van den Brink, E. C. M. y Yannai, E. (eds.), In Quest of Ancient Settlements and Landscapes: Archaeological Studies in Honour of Ram Gophna. Tel Aviv: Ramot, 45-56.

»Getzov, N. (2006). The Tel Bet Yerah Excavations, 1994-1995. Jerusalén: Israel Antiquities Authority.

»Getzov, N. (2016). Restos del período Calcolítico tardío en Einot Kaukab en la Galilea occidental, en: 'Atiqot 87: $1^{*}-41^{*}$ (hebreo con resumen en inglés).

" Getzov, N. y Barzilai, O. (2011). Mishmar Ha-'Emeq (el-Ghab et-Tahta), en: Hadashot Arkheologiyot - Excavations and Surveys in Israel 123. https://www. hadashot-esi.org.il/report_detail_eng.aspx?id=1896\&mag_id=118 (accedido 7/3/2021)

» Getzov, N., Vardi, J. y Marom, N. (en prensa). Ard el-Samra: un asentamiento proto-histórico en los márgenes de la planicie de Akko, en: 'Atiqot (hebreo).

"Golani, A. (2003). Salvage Excavations at the Early Bronze Age Site of Qiryat Ata. Jerusalén: Israel Antiquities Authority.

» Golani, A. (2014). Rescue Excavations at the Early Bronze Age Site of Qiryat Ata, Area N, en: 'Atiqot 79: 11-44.

" Golani, A., Storchan, B. y Eirikh-Rose, A. (2018). The Chalcolithic and Early Bronze Age IB Site of Beqo'a, en: 'Atiqot 90: 9-54.

» Gophna, R. (1996). Excavations at Tel Dalit. An Early Bronze Age Walled Town in Central Israel. Tel Aviv: Ramot.

»Goren, Y. (1991) Beginning of Pottery Production in Israel. Tesis de doctorado. Jerusalén: The Hebrew University of Jerusalem (hebreo).

» Goren, Y. y Goring Morris, A.N. (2008). Early Pyrotechnology in the Near East: Experimental Lime-Plaster Production at the Pre-Pottery Neolithic B Site of Kfar HaHoresh, Israel, en: Geoarchaeology 23 (6): 779-798.

"Goši , M. y Gilead, I. (2015). Unveiling Hidden Rituals: Ghassulian Metallurgy of the Southern Levant in Light of the Ethnographical Record, en: Rosińska-Balik, K., Ochał-Czarnowicz, A., Czarnowicz, M. y Dębowska-Ludwin, J. (eds.), Copper and Trade in the South-Eastern Mediterranean Trade routes of the Near East in Antiquity. Oxford: BAR, 25-38.

"Greenberg, R., Rotem Y. y Paz, S. (2013). The Earliest Occupation at Tel Bet Yerah, en: Tel Aviv 40: 197-225.

» Greenberg, R., Eisenberg, E., Paz, S. y Paz, Y. (2006). Beth Yerah, The Early Bronze Age Mound. Vol. I, Excavations Reports, 1933-1986. Jerusalén: Israel Antiquities Authority.

"Kaplan, J. (1952). Una tumba de la Edad del Bronce Antiguo descubierta en Tel Aviv, en: Yediot 16 (3-4): 25 (hebreo).

» Kaptijn, E. (2009). Life on the Watershed. Reconstructing Subsistence in a Steppe Region Using Archaeological Survey: A Diachronic Perspective on Habitation in the Jordan Valley. Leiden: Leiden University.

» Kempinski, A. y Gilead, I. (1991). New Excavations at Tel Erani: A Preliminary Report of the 1985-1988 Seasons, en: Tel Aviv 18: 164-191.

" Lamberg- Karlovsky, C. C. (2009). Vere Gordon Childe and the Concept of 
Revolution, en: Shea, J. J. y Lieberman, D. L. (eds.), Transitions in Prehistory. Essays in Honor of Ofer Bar-Yosef. Harvard: Harvard University Press, 445-467.

"Levy, T. E. (2007). Journey to the Copper Age. San Diego, CA: The Museum of San Diego.

» Marquet-Krause J. (1949). Les fouilles de ‘Ay (Et-Tell). París: Librairie Orientaliste Paul Geuthner.

» Marx, K. (1970 [1859]). A Contribution to the Critique of Political Economy. Nueva York: International Publishers.

" Milevski, I. (2011). Centros urbanos, periferia e intercambio en el Levante meridional durante la Edad del Bronce Antiguo, en: Eretz-Israel 30: 317-327 (hebreo con resumen en inglés).

» Milevski, I. (2013). The Transition from the Chalcolithic to the Early Bronze Age of the Southern Levant in Socio-Economic Context, en: Paléorient 39 (1): 193-208.

»Milevski, I. (2016). Intercambio de productos en el Levante meridional durante el Bronce Antiguo. Una perspectiva marxista. Barcelona: Universidad Pompeu Fabra.

» Milevski, I. (2019). Las costumbres de enterramiento de la prehistoria tardía en el Levante meridional y el concepto "modos funerarios" como instrumento de investigación, en: Varf, D., Abadi-Reis, Y., Lehman, G. y Vainstub, D. (eds.), Enterramientos y culto en la Sefela y el Negev a través de los tiempos. Beersheva: Israel Antiquities Authorities y Ben-Gurion University of the Negev, 9-30 (hebreo).

» Milevski, I. y Getzov, N. (2014). Ein Zippori. Preliminary Report, en: Hadashot Arkheologiyot- Excavations and Surveys in Israel 126. http://www.hadashot-esi. org.il/Report_Detail_Eng.aspx?id=13675 (accedido 15/12/2014)

» Milevski, I. y Horwitz, L. K. (2019). Domestication of the Donkey (Equus asinus) in the Southern Levant: Archaeozoology, Iconography and Economy, en: Kowner, R., Bar-Oz, G., Biran, M., Meir, S. y Shelach-Lavi, G. (eds.), Animals and Human Society in Asia: Historical, Cultural and Ethical Perspectives. Cham: Palgrave Macmillan, 93-148.

» Milevski, I., Liran, R. y Getzov, N. (2014). The Early Bronze Age Town of 'En Zippori in the Galilee (Israel), en: Antiquity Project Gallery 339. http://www. antiquity.ac.uk/projgall/milevski339/ (accedido 5/3/2021)

» Milevski, I., Yegorov, E., Aladjem, E. y Pasternak, M. D. (2016). Salvage Excavation at Tel Erani, Areas P to U, en: Ciałowicz, K., Yekutieli, Y. y Czarnowicz, M. (eds.), Tel Erani I. Preliminary Report of the 2013-2015 Excavations. Cracovia: Wydawnictwo Alter, 45-58.

» Milevski, I., Getzov, N. y Paz, Y. (en prensa). Uneven and Combined: The Synchronization of the Early Bronze Age I and the First Urbanization of the Southern Levant, en: Adams, M. J., Höfflmayer, F. y Roux, V. (eds.), Transitions during the Early Bronze Age in the Levant. Münster: Zaphon.

» Milevski, I., Campagno, M., Gandulla, B., Jaruf, P., Daizo, M. B., Czarnowicz, M., Ochał-Czarnowicz, A., Karmowski, J., Yegorov, D., Cohen-Sasson, E. y Yekutieli, Y. (2019). Tel Erani, Israel: Reporte de la campaña arqueológica de 2018 y sus antecedentes, en: Revista del Instituto de Historia Antigua Oriental 20: 5-22.

" de Miroschedji, P. (1988). Yarmouth 1. Rapport sur les trois campagnes de fouilles à Tel Yarmouth (Israël), 1980-1982. París: CNRS. 
» de Miroschedji, P. (2012-2013). L'apparition des palais au Levant méridional au Bronze ancien et sa signication, en: Michel, C. (ed.), De la maison à la ville dans l'Orient Âncient: bâtiments publics et lieux de pouvoir. Nanterre: CNRS, 95-101.

»Namdar, D., Amrani, A., Getzov, N. y Milevski, I. (2015). Olive Oil Storage during the Fifth and Sixth Millennia BC at Ein Zippori, Northern Israel, en: Israel Journal of Plant Sciences 62 (1-2): 65-74.

»Papadopoulos, C., Hamilakis, Y. y Kparissi-Apostolika, N. (2015). Light in a Neolithic Dwelling: Building 1 at Koutroulou Magoua Greece, en: Antiquity 89 (347): 1034-1050.

»Paz, Y., Elad, I., Milevski, I. y Getzov, N. (2018). Geometric Motifs and Cultic Scenes on Cylinder Seal Impressions found in Early Bronze IB Urban Centers of the Southern Levant, en: Ugarit-Forschungen 49: 285-298.

»Pettitt, P., Leluschko, S. y Sakamoto, T. (2017). Light, Human Evolution, and the Palaeolithic, en: Papadopoulos, C. y Moyes, H. (eds.), The Oxford Handbook of Light in Archaeology. Oxford: Oxford University Press. 10.1093/ oxfordhb/9780198788218.013.1 (accedido 7/9/2021)

»Prag, K. (1991). Preliminary Report on the Excavations at Tell Iktanu and Tell alHammam, Jordan, 1990, en: Levant 2: 55-66.

»Prag, K. (2000). Tell Iktanu, South Jordan Valley: Early Bronze Age I Ceramics, en: Philip, G. y Baird, D. (eds.), Ceramics and Change in the Early Bronze Age of the Southern Levant. Sheffield: Sheffield Academic Press, 91-99.

»Regev, J., de Miroschedji, P. y Boaretto, E. (2012a). Early Bronze Age Chronology: Radiocarbon Dates and Chronological Models from Tel Yarmuth (Israel), en: Radiocarbon 54 (3-4): 505-524.

»Regev, J., de Miroschedji, P., Greenberg, R., Braun, E., Greenhut, Z. y Boaretto, E. (2012b). Chronology of the Early Bronze Age in the Southern Levant: New Analysis for a High Chronology, en: Radiocarbon 54 (3-4): 525-566.

» Regev, J., Paz, S., Greenberg, R. y Boaretto, E. (2019). Radiocarbon Chronology of the EB I-II and II-III Transitions at Tel Bet Yerah, and Its Implications for the Nature of Social Change in the Southern Levant, en: Levant 51: 54-75.

»Rosenzweig, G. (2020). Absolute Chronology for the Ghassulian Culture: Case Studies from Ashkelon Agamim and Tel Sheva, and Their Implication on the Overall Chronological Framework. Tesis de maestría. Rehovot: Weizmann Institute of Science.

»Rotem, Y. (2012). The Early Bronze Age IB Pottery from Strata M-3 and M-2, en: Mazar, A. (ed.), Excavations at Beth-Shean 1989-1996, Volume IV, The 4th and 3rd Millennia BCE. Jerusalén: Israel Exploration Society, 123-235.

"Rotem, Y. (2015). El valle del Jordán medio durante la Edad del Bronce Antiguo I y la transición al Bronce Antiguo II: Patrones y procesos en la sociedad aldeana compleja. Tesis de doctorado. Tel Aviv: Tel Aviv University (hebreo).

"Schaub, R. T. (2000). Terminology and Typology of Carinated Vessels of the Early Bronze Age I-Il of Palestine, en: Stager, L. E., Greene, J. A. y Coogan, M. D. (eds.), The Archaeology of Jordan and Beyond: Essays in Honor of James A. Sauer. Winona Lake, IN: Eisenbrauns, 444-464.

"Smith, A. (1904 [1776]). An Inquiry into the Nature and Causes of the Wealth of Nations, 2 vols. Londres: Methuen.

"Sussman V. (1985). Lighting the Way Through History: The Evolution of Ancient 
Oil Lamps, en: Biblical Archaeology Review 11 (2): 42-56.

"Ussishkin, D. (2014). The Sacred Area of Early Bronze Megiddo: History and Interpretation, en: Bulletin of the American Schools of Oriental Research 375: 78-104.

»West, I. (2017). Industrializing Light: The Development and Deployment of Artificial Lighting in Early Factories, en: Papadopoulos, C. y Moyes, H. (eds.), The Oxford Handbook of Light in Archaeology. Oxford: Oxford University Press. DOI: 10.1093/oxfordhb/9780198788218.013.23 (accedido 7/9/2021)

» Yannai, E. (2016). 'En Esur ('Ein Asawir) II, Excavations at The Cemeteries. Jerusalén: Ostracon-Israel Antiquitites Authority.

» Yaroshevich, A. (2016). En Zippori. Preliminary Report, en: Hadashot Arkheologiyot - Excavations and Surveys in Israel 128. https://www.hadashot-esi. org.il/report_detail_eng.aspx?id=24979\&mag_id=124 (accedido 5/3/2021)

"Yeivin, S. (1961). First Preliminary Report on the Excavations at Tel "Gat" (Tell Sheykh Ahmed el-Areyny): Seasons 1956-1958. Jerusalén: Department of Antiquities of Israel. 
